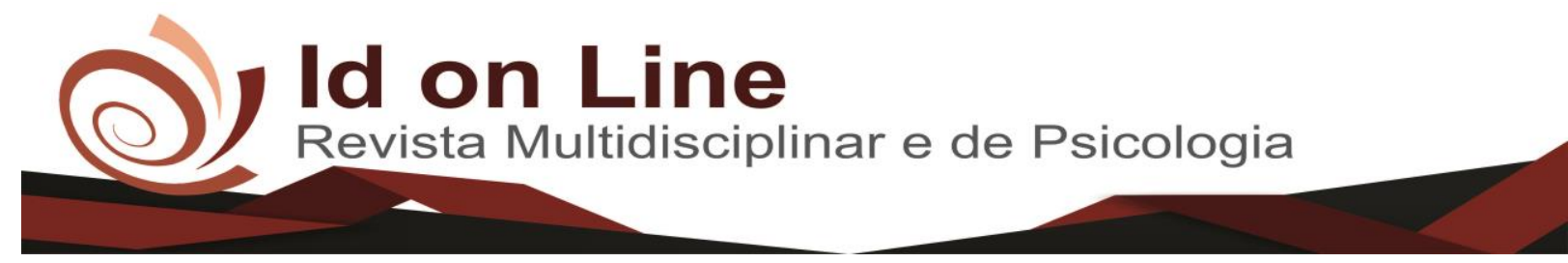

Comment

\title{
Disfunção do Trato Urinário Inferior em Crianças: Revisão de Literatura
}

\author{
Geniefesson Leandro da Silva Feitoza ${ }^{1}$, Lucas Benevides Maia ${ }^{1}$, \\ Débora Laésia Saraiva Ribeiro', Lucas Leimig Telles Parente', \\ Rodrigo Emmanuel Leimig Telles Parente' ${ }^{\text {, Maria Valéria Leimig Telles }}{ }^{2}$
}

\begin{abstract}
Resumo: A disfunção do trato urinário inferior (DTUI) é um quadro comum em crianças, manifestando-se principalmente por incontinência urinária (IU), infecção do trato urinário (ITU), refluxo vesicoureteral (RVU) e constipação. A Sociedade Internacional de Continência (ICS) define a IU como a perda involuntária de urina em maiores ou igual a 5 anos, sendo considerado um sintoma de armazenamento. É uma doença multipatogênica envolvendo o sistema nervoso central, ritmo circadiano (sono/vigília) e alterações funcionais da bexiga. O sexo feminino tem mais propensão a sofrer com IU devido a algumas diferenciações anatômicas em relação ao sexo masculino. Metodologia: Revisão bibliográfica a partir das bases de dados LILACS, MedLine, Scielo e Pubmed, realizada nos meses de julho e agosto de 2018. As palavras-chaves foram urinary incontinency, urology, chil e enuresis e os critérios de inclusão foram idioma espanhol, inglês e português publicados de 2009 a 2018 , relacionados com o tema do estudo, e os de exclusão foram artigos anteriores a 2009 e os artigos não relacionados ao tema. Dos 57 artigos selecionados, foram utilizados 17. Discussão: A DTUI pode apresentar-se de diversas formas, com a combinação de múltiplos sinais e sintomas, por vezes acompanhada também de alterações do trato gastrointestinal. Essa apresentação clínica pode alterar de forma importante o convívio social dessas crianças, provocando comorbidades psicológicas, o que afeta diretamente o desenvolvimento desses pacientes. O diagnóstico passa por um olhar atento e uma anamnese adequada, buscando identificar essas alterações miccionais, podendo estar associado a exames complementares. $\mathrm{O}$ tratamento deve ser individualizado, iniciando com alterações comportamentais e informações sobre a doença até um tratamento farmacológico quando for adequado. Conclusão: Diante da repercussão clínica e psicológica de tal patologia, faz-se importante um diagnóstico precoce e um tratamento adequado, evitando mais transtornos e melhorando o aspecto social e psicológico do paciente e de seus familiares.
\end{abstract}

Palavras-chave: Trato urinário, Saúde da criança, vesicoureteral, constipação.

\section{Lower Urinary Tract Dysfunction in Children: A Literature Review}

\begin{abstract}
Low urinary tract dysfunction (LUT) is a common condition in children, manifesting mainly through urinary incontinence (UI), urinary tract infection (UTI), vesicoureteral reflux (VUR) and constipation. The International Continuum Society (ICS) defines UI as involuntary loss of urine over 5 years and is considered a storage symptom. It is a multipathogenic disease involving the central nervous system, circadian rhythm (sleep/wake) and functional changes of the bladder. The female sex is more likely to suffer from UI due to some anatomical differentiations in relation to the male sex. Methodology: Bibliographic review from LILACS, MedLine, Scielo and Pubmed databases, carried out in July and August 2018. The keywords were urinary incontinence, urology, chil and enuresis, and the inclusion criteria were Spanish language, English and Portuguese published from 2009 to 2018, related to the theme of the study, and those of exclusion were articles prior to 2009 and articles not related to the topic. LUT can be present in a variety of ways, with a combination of multiple signs
\end{abstract}

\footnotetext{
${ }^{1}$ Faculdade de Medicina Estácio de Juazeiro do Norte, Ceará, Brasil.

${ }^{2}$ Médica. Doutorado em Neurologia / Neurociências pela Universidade Federal de São Paulo, UNIFESP. Professora da Faculdade de Medicina Estácio de Juazeiro do Norte, Ceará, Brasil. Contato: valerialeimigtelles@ hotmail.com.
} 
and symptoms, sometimes accompanied by changes in the gastrointestinal tract. This clinical presentation can significantly alter the social life of these children, provoking psychological comorbidities, which directly affects the development of these patients. The diagnosis goes through a close look and an adequate anamnesis, seeking to identify these voiding changes, and may be associated with complementary tests. Treatment should be individualized, starting with behavioral changes and information on the disease until a pharmacological treatment when appropriate. Thus, in view of the clinical and psychological repercussion of such pathology, it is important to have an early diagnosis and an appropriate treatment, avoiding more disorders and improving the social and psychological aspect of the patient and their relatives.

Keywords: Urinary tract, Child's health, vesicoureteral, constipation.

\section{Introdução}

A disfunção do trato urinário inferior (DTUI) ou LUTS (do inglês Lower Urinary Tract Symptoms) é uma expressão que caracteriza todos os distúrbios miccionais, seja na fase de enchimento ou na fase esvaziamento, não havendo patologia neurológica ou obstrução do trato urinário. Em crianças, é um quadro comum, manifestando-se principalmente por incontinência urinária (IU), infecção urinária, refluxo vesicoureteral e constipação (BALLEK; MCKENNA, 2010). Entre essas queixas, destaca-se a IU, afetando muitas crianças em idade escolar, com uma prevalência entre 6,3 e15,0\% entre crianças (CUPELLI et al.,2014).

A Sociedade Internacional de Continência (ICS) definiu a IU como o relato de qualquer perda involuntária de urina em pessoas com idade maior igual a 5 anos. Podemos subdividir a incontinência em contínua ou intermitente e em diurna ou enurese. A enurese, por sua vez, é classificada de acordo com o quadro clínico do paciente: sem outros sintomas urinários e sem disfunção da bexiga, é definida como enurese monossintomática (EM); com a associação de pelo menos um outro sintoma urinário é classificado como enurese não monossintomática (ENM). Uma vez que os sintomas LUTS cessam, a enurese muda de não monossintomática para monossintomática (AUSTIN et al. 2014).

Johan e colaboradores (2012) definiram a fisiopatologia da enurese como complexa, pois envolve o sistema nervoso central, ritmo circadiano e alterações funcionais da bexiga.

Eliminações disfuncionais é outro termo que pode ser usado quando coexistem sintomas de LUTS associados com disfunção intestinal (VASCONCELOS et al. 2013). 
Para Franco (2011), há cada vez mais estudos relacionando os sintomas LUTS com disfunção intestinal. Essa relação é explicada pelo fato de o trato geniturinário e o trato gastrointestinal dividerem a mesma embriologia, região anatômica e ramos nervosos. Outra região comum a ambos os sistemas, é o assolho pélvico, atuando no controle do ato miccional e da evacuação. Sintomas comuns incluem urgência miccional, enurese noturna e incontinência fecal (CUPELLI et al.,2014).

A prevalência dos sintomas de DTUI apresenta-se de forma variável na literatura, com trabalhos que variam entre 2 e $25 \%$. Essa variação se deve principalmente às diferentes terminologias usadas pelos pesquisadores (VAZ, G.T. et al.,2011).

No Brasil, observou-se prevalência de sintomas miccionais de 22,8\% em crianças de 3 a 9 anos, sendo 10,5\% para os meninos e 33,8\% para as meninas; em crianças de 6 a 12 anos, a prevalência foi de $21,8 \%$, sendo $10,7 \%$ meninos e $31,1 \%$ meninas, se assemelhando á epidemiologia mundial (BURNS, et al. 2017).

\section{Metodologia}

O presente estudo trata-se de uma revisão bibliográfica a partir das bases de dados Literatura Latino-Americana e do Caribe em Ciências da Saúde (LILACS), Literatura Internacional em Ciências da Saúde (MEDLINE), Scientific Electronic Library Online (SciELO), Cochane Library e Pubmed. realizada nos meses de julho e agosto de 2018.

As palavras-chaves utilizadas foram "urinary incontinency”, "urology”, "child” $e$ "enuresis", e os critérios de inclusão compreendem artigos nos idiomas espanhol, inglês e português publicados no período entre 2009 e 2018, relacionados com o tema do estudo, e os critérios de exclusão foram artigos anteriores a 2009 e os artigos não relacionados ao tema. A busca apresentou 57 resultados, dos quais apenas 17 foram selecionados após a aplicação dos critérios de inclusão e exclusão. 


\section{Discussão}

Nos neonatos, o reflexo sacral da medula espinal é o único responsável por eliminar a urina. Com o passar do tempo, os reflexos primitivos começam a ser suprimidos, o que leva ao aumento da capacidade vesical, reduzindo a frequência miccional. Por volta do segundo ao quarto ano de idade, inicia-se o desenvolvimento da sensação consciente da bexiga e, nesse momento, a criança inicia o autocontrole do ato miccional (REYNARD; BREWSTER; BIERS, 2013).

A ICCS classifica esses distúrbios, conforme descrito a seguir:

\section{Bexiga hiperativa (BHA)}

Para Austin e colaboradores (2014), a BHA é caracterizada por urgência urinária, normalmente acompanhada de micções frequentes e noctúria, com ou sem IU, na ausência de infecção do trato urinário (ITU) ou outra patologia. É o tipo mais prevalente da DTUI, e está associada à hiperatividade detrusora. Crianças acometidas se utilizam de algumas manobras, como cruzar as pernas, apertar a genitália com a mão, sentar-se por cima da perna, com o objetivo de manter-se continente.

\section{Adiamento miccional}

São crianças com incontinência diurna que utilizam manobras para contenção. Associado à redução no número de micções, há sensação de urgência e redução da ingesta de líquidos, a fim de evitar perdas urinárias. Nessas crianças, há elevada incidência de comorbidades psicológicas ou distúrbios comportamentais (VASCONCELOS et al., 2013).

\section{Bexiga hipoativa:}

Uma condição em que a frequência urinária é baixa e há necessidade de aumentar a pressão intra-abdominal para iniciar, manter ou completar a micção. As crianças podem 
apresentar frequência reduzida de micções. Pode acontecer devido a uma capacidade de contração diminuída do músculo detrusor e/ou uma grande complacência vesical (NEVEUS et al., 2006).

\section{Micção disfuncional}

Disfunção que ocorre apenas na fase de esvaziamento. Os músculos do assoalho pélvico se contraem durante o ato miccional, impedindo-a de acontecer. Para o diagnóstico, é preciso uma avaliação da urofluxometria livre (início do estudo urodinâmico) com demonstração de curvas com fluxo intermitente (interrompido) ou staccato (flutuante), associado ao exame US para avaliar o resíduo pós-miccional. Os sintomas podem variar desde uma micção incoordenada até o quadro mais grave de dissinergia vésico-perineal. Pode resultar em lesão do trato urinário e RVU. Essa expressão é usada para pacientes neurologicamente íntegros (REYNARD; BREWSTER; BIERS, 2013).

\section{Obstrução}

Ocorre um impedimento ao fluxo urinário durante a micção. É caracterizado por um aumento da pressão do músculo detrusor com um fluxo urinário diminuído (NEVEUS et al., 2006).

\section{Incontinência por estresse}

É a liberação de urina durante o esforço físico ou aumento da pressão intra-abdominal, como ocorre no espirro e na tosse (NEVEUS et al., 2006). 


\section{Incontinência do riso}

Refere-se à perda urinária durante o riso. Uma teoria é que o riso possa induzir a diminuição do tônus de forma generalizada com relaxamento uretral, levando à incontinência urinária (CHANDRA et al., 2002). A função da bexiga se mantém preservada quando não há riso (AUSTIN et al., 2014).

\section{Refluxo vaginal}

Ocorre quando meninas que possuem controle da micção apresentam episódios de perda urinária em pequenas quantidades logo após urinar (NEVEUS et al., 2006).

\section{Frequência urinária diurna aumentada}

Aumento da frequência miccional em que pequenos volumes são liberados a cada micção. O paciente não apresenta outros sintomas diurnos ou noturnos associados. Excluir outros diagnósticos, como diabetes tipo I, diabetes insipidus nefrogênica, ITU ou síndrome viral (NEVEUS et al., 2006).

\section{Enurese noturna}

A enurese é tanto um sintoma quanto uma condição de incontinência intermitente que ocorre durante o período em que a criança dorme (AUSTIN et al., 2014). De acordo com o DSM-5 e CID-10, enurese é significativa se ocorre > 1 episódio por mês e uma frequência de 3 episódios ao longo de 3 meses. Propõe-se, ainda, qualificar como frequente (4 episódios por semana) ou infrequente ( $<4$ episódios por semana).

Enurese monossintomática é definida como enurese sem outros sintomas LUTS (excluindo noctúria). Enurese não monossintomática define a presença de outros sintomas LUTS. Enurese primária é quando o paciente permanece seco por um período inferior a 6 meses, e na secundária ocorre uma recidiva dos sintomas após 6 meses de continência (NEVEUS et 
al., 2006). Predisposição familiar, fatores psicológicos, ITU e constipação também contribuem para a enurese noturna.

\section{Disfunção da bexiga e intestino (BBD, do inglês bladder/bowel dysfunction)}

A BBD inclui anormalidades no trato urinário inferior podendo incluir padrões intestinais anormais como constipação e encoprese, predispondo crianças a ITU, IU e desfechos com RVU (BURGERS, 2013). Consiste em múltiplos sintomas, como IU diurna e enurese, alteração da frequência urinária, urgência miccional e constipação. O quadro clinico pode variar e o diagnóstico é frequentemente por exclusão. Há pouca padronização para diagnosticar de forma confiável essa disfunção (DRZEWIECKI, 2012).

\section{Comorbidades associadas}

Além da correlação entre sintomas urinárias e gastrointestinais, outra associação bem documentada é entre sintomas urinários, em especial a enurese, e comorbidades psicológicas (ARLEN, 2014). Especialmente os distúrbios do comportamento, que afetam de 20 a $40 \%$ das crianças com enurese e 30 a 40\% com incontinência diurna (BAUER, 2012).

Esses pacientes exibem mais problemas comportamentais e/ou psicológicos, como ansiedade, déficit de atenção, hiperatividade ou transtornos de conduta. Mesmo aqueles com enurese monossintomática frequentemente exibem taxas mais altas (FRANCO, 2011). Ainda não está claro se a incontinência é um resultado de problemas comportamentais, ou o inverso (CUPELLI et al.,2014).

Vaz e colaboradores (2011) realizaram um estudo na cidade de Belo Horizonte, onde encontrou aproximadamente um quarto das crianças com sintomas miccionais relatando eventos estressantes em suas vidas. Por isso, a importância de uma observação atenta (CHING et al., 2015). 


\section{Diagnóstico}

Uma história completa e um exame físico minucioso são as principais ferramentas para avaliação de crianças com disfunção de LUTS. Observar a criança para manobras de contenção, expressões de urgência ou outros problemas comportamentais (AUSTIN et al., 2014).

A investigação detalhada dos hábitos miccionais e intestinais pode ser feita usando um diário miccional e/ou questionários estruturados. $\mathrm{O}$ ambiente social da criança e o desenvolvimento geral e comportamental também devem ser formalmente avaliados e registrados. Análise e cultura de urina é suficiente para excluir infecção. Se possível, deve observar-se a criança durante a micção (ABRAMS et al., 2010).

O Diário das eliminações deve ser usado em maiores de 5 anos para aferir a capacidade da bexiga da criança. Medição do volume com um mínimo de 3 a 4 dias para melhor precisão dos resultados. Qualquer vazamento de urina durante o dia, bem como volumes de ingestão de fluidos também devem ser registrados (JOHAN et al.,2012).

A urofluxometria é um exame simples que estuda as características do fluxo urinário. Para melhor sensibilidade, o paciente deve ter controle esfincteriano, e o volume total urinado deve corresponder a pelo menos $50 \%$ da capacidade vesical esperada (CVE) para a idade (AUSTIN et al., 2014).

Esse exame pode ser associado com a eletromiografia dos músculos do assoalho pélvico, podendo evidenciar uma hiperatividade desses músculos ou uma incoordenação vesicoesfincteriana. É importante quantificar o resíduo pós-miccional através do US após o exame (FRANCO, 2012).

Como método de imagem, o exame fundamental é a ultrassonografia de vias urinárias (AUSTIN et al., 2014). Só ela é capaz de medir o volume da bexiga pré e pós-miccional, o volume de urina residual, espessura da parede da bexiga, contrações involuntárias, comportamento da pelve e ureter durante o período de enchimento, esvaziamento vesical e alterações anatômicas das vias urinárias (VASCONCELOS et al., 2013). Pode avaliar-se o reto, seu diâmetro transversal, presença de fezes na ampola retal, que sugerem ou não constipação e/ou impactação fecal. O diâmetro do reto de forma isolada não é um preditor de constipação e/ou impactação fecal (FRANCO et al., 2013). 
O estudo urodinâmico é considerado o padrão-ouro. A cistometria faz a avaliação da fase do enchimento da bexiga, ela analisa a relação entre o volume e a pressão vesical. São registrados parâmetros, como a sensibilidade vesical, a atividade detrusora, a complacência e a capacidade vesical (CHAPPLE; MACDIARMID; PATEL, 2009). Ele é capaz de diagnosticar diversos padrões de alteração funcional, classificando os casos em hiperatividade detrusora e/ou a micção disfuncional por incoordenação vesicoesfincteriana ou obstrução (CHAPPLE; MACDIARMID; PATEL, 2009).

A Uretrocistografia miccional deve ser solicitada em crianças de ambos os sexos com ITU de repetição e com hidronefrose detectada ao exame ultrassonográfico de vias urinárias com o objetivo de detectar alterações morfofuncionais da bexiga, refluxo vesicoureteral ou alterações anatômicas da bexiga e uretra (HOEBEKE et al., 2010).

\section{Tratamento}

O tratamento inicial deve ser sempre baseado em técnicas comportamentais (uroterapia). Essa técnica atua por meio da informação e desmitificação, informando aos pacientes sobre a doença, as modificações comportamentais importantes, a alteração do estilo de vida e desfazendo conceitos preconcebidos.

Outra alternativa é a terapia de alarme com um grau A de recomendação do ICS, para enurese, no entanto, pode ser usado também durante o dia. Esse método funciona com a colocação de um sensor na fralda ou roupa do paciente que dispara sempre que entra em contato com a urina, fazendo que a criança perceba e cesse o ato miccional, devendo ir ao banheiro esvaziar a bexiga. $\mathrm{O}$ alarme deve ser usado de forma contínua por pelo menos de 2 a 3 meses.

Há também a terapia de neuromodulação transcutânea, com a estimulação do nervo tibial posterior fornecendo neuromodulação para o assolho pélvico através do plexo do nervo sacral. É uma alternativa interessante que vem ganhando espaço.

O tratamento farmacológico vem em ultimo caso, após a ineficácia de todos os outros comportamentais e de neuromodulação. A oxibutinina é a droga de escolha, sendo a principal medicação prescrita e aprovada pelo Food And Drug Administration (FDA). Sua ação anticolinérgica tem como principal função diminuir a hiperatividade vesical. A desmopressina 
pode ser utilizada para reduzir o volume urinário produzido durante a noite, tornando-se uma opção na enurese. Os alfa-bloqueadores, podem ser utilizados como drogas de segunda linha, a doxazosima demonstrou resultados satisfatórios.

\section{Conclusões}

A DTUI pode apresentar-se de diversas formas, desde forma monossintomática a com múltiplos sinais e sintomas. Na maioria das vezes, a queixa não parte da criança ou dos seus pais, que podem considerar o quadro apresentado como algo normal para idade, principalmente quando acomete crianças. Por isso, faz-se necessária uma anamnese detalhada e um questionamento específico para esse sistema, na busca de um diagnóstico adequado e precoce, evitando mais transtornos ao paciente e melhorando o aspecto social e psicológico do paciente e de seus familiares.

\section{Referências}

ABRAMS, K.E. et al. Fourth International Consultation on Incontinence Recommendations of the International Scientific Committee: Evaluation and Treatment of Urinary Incontinence, Pelvic Organ Prolapse, and Fecal Incontinence. Neurourol Urodyn. V.29, Pág. 213-240, 2010.

\section{AMERICAN PSYCHIATRIC ASSOCIATION. Manual de diagnóstico e estatística de distúrbios mentais DSM- V. São Paulo: ARTMED, 2015.}

ARLEN, A.M. et al. Phantom Urinary Incontinence in Children With Bladder-bowel Dysfunction. Urology. Vol. 84, Pág.685-688, 2014.

AUSTIN, P.F. et al. The standardization of terminology of lower urinary tract function in children and adolescents: update report from the Standardization Committee of the International Children's Continence Society. The Journal Of Urology. V.191, Pág.1865-1878, 2014.

BALLEK, N.K.; MCKENNA P.H. Lower urinary tract dysfunction in childhood. Uro Clin North Am. V.37,Pág.215-228, 2010.

BAUER, S. B., AUSTIN, P. F., RAWASHDEH, Y. F. et al. International Children's Continence Society's recommendations for initial diagnostic evaluation and follow-up in 
congenital neuropathic bladder and bowel dysfunction in children. Neurourol Urodyn, V.31, Pág. 610, 2012.

BURGERS, R. et al. Functional defecation disorders in children with lower urinary tract symptons. J Urol. V.189, Pág. 1886-1891, 2013.

BURNS, D.A. et al. Tratado de Pediatria: Sociedade Brasileira de Pediatria. 4. ed. Barueri, SP : Manole, V.1,Pág. 1119-1125, 2017.

CHANDRA, M.; SAHARIA, R.; SHI, Q.; HILL, V. Giggle incontinence in children: a manifestation of detrusor instability. J Urol. V.168, Pág.2184-2187, 2002.

CHAPPLE, C.R.; MACDIARMID S.A.; PATEL A. Urodynamics made easy. 3. ed. Espanha: Elsevier Churchill Livingtone. Cap. 2, Pág. 7-19, 2009.

CHING C.B. et al. Bullying and lower urinary tract symptoms: why the pediatric urologist should care about school bullying. J Urol. V.193,Pág.650-654, 2015.

CUPELLI, E.T.; ESCALLIER L.; GALAMBOS, N.; XIANG, S.; FRANCO, I. Sensory processing differences and urinary incontinence in school-aged children. Journal of Pediatric Urology V.10,Pág.880-885, 2014.

DRZEWIECKI, B.A. et al. Use of validated Bladder/Bowel Dysfunction questionare in the Pediatric urology Setting. J Urol. V.188, Pág. 1578-1583, 2012.

FRANCO I. Neuropsychiatric disorders and voiding problems in children. Curr Urol Rep. Vol.12, Pág.158-165, 2011.

HOEBEKE P, BOWER W, COMBS A, DE JONG T, YANG S. Diagnostic evaluation of children with daytime incontinence. J Urol. V.183, Pág. 699-703, 2010.

JOHAN V.W. et al. Practical consensus guidelines for the management of enuresis. Eur $\mathbf{J}$ Pediatr. V.171, Pág.971-983, 2012.

NEVÉUS T.V. et al. The Standardization Of Terminology Of Lower Urinary Tract Function In Children And Adolescents: Report From The Standardization Committee Of The International Children's Continence Society. J Urol. Vol.176, Pág.314-324, 2006.

ORGANIZAÇÃO MUNDIAL DA SAÚDE. CID-10 Classificação Estatística Internacional de Doenças e Problemas Relacionados à Saúde. 10. ed. São Paulo: Universidade de São Paulo, 1997.

REYNARD, J.; BREWSTER, S.; BIERS, S. Oxford Handbook Of Urology. 3. ed. United Kingdom. Oxford University Press, 2013. 
VASCONCELOS, M.M. et al. Lower urinary tract dysfunction- acommon diagnosis in the pediatrics practice. Jornal Brasileiro de Nefrologia. V.35,Pág.57-64, 2013.

VAZ, G.T. et al. Prevalence of lower urinary tract symptoms in school-age children. Pediatr Nephrol. V.27,Pág.597-603, 2011.

\section{Como citar este artigo (Formato ABNT):}

FEITOZA, Geniefesson Leandro da Silva; MAIA, Lucas Benevides; RIBEIRO, Débora Laésia Saraiva; PARENTE, Lucas Leimig Telles; PARENTE, Rodrigo Emmanuel Leimig Telles; TELLES, Maria Valéria Leimig. Disfunção do Trato Urinário Inferior em Crianças: Revisão de Literatura. Id on Line Rev.Mult. Psic., 2018, vol.12, n.42, Supl. 1, p. 133-144. ISSN: 1981-1179.

Recebido: 29/10/2018;

Aceito: 03/11/2018 